\title{
Factores asociados a mortalidad en pacientes Covid- 19 en un Hospital del norte de Perú
}

\author{
Factors associated with mortality in Covid-19 \\ patients in a Hospital in northern Peru
}

Saúl Murrugarra-Suarez ${ }^{1,2, a, b}$, Miryam Lora-Loza ${ }^{3,4, c, d}$, José Cabrejo-Paredes ${ }^{5}$ e,f, Luis Mucha-Hospinal ${ }^{6, g, h}$, Hugo Fernandez-Cosavalente ${ }^{7,8, i, j}$

\begin{abstract}
RESUMEN
Objetivo. Determinar los factores de riesgo sociodemográficos y clínicos relacionados con la mortalidad en pacientes COVID-19 en un Hospital del norte de Perú. Material y Métodos: la investigación se llevó a cabo en un Hospital del norte de Perú. La población la constituyeron 208 Historias Clínicas de pacientes COVID-19 atendidos entre Marzo a Julio del 2020. La variable independiente fueron los factores sociodemográficos tales como la edad, sexo y el estado civil y los factores clínicos como antecedentes epidemiológicos, los factores de riesgo (comorbilidades) y el estado de salud y la variable dependiente fue la mortalidad Covid-19. Resultados: La mortalidad COVID-19 alcanzó al 46,20 \% en pacientes mayores 65 años (51,90\%), de sexo masculino (60,40\%) y casados (65,40 \%), con antecedentes epidemiológicos (37,5\%), y factores de riesgo (comorbilidad) un 74,50\%, principalmente asociados con hipertensión arterial (8,2\%), obesidad $(3,4 \%)$ y diabetes mellitus II $(1,9 \%)$, y comorbilidades indeterminadas (Otras) un 6,7\%. No se presentó mortalidad en los pacientes cuyo estado de salud era Estable, mientras que los pacientes con estado de salud reservado y fallecieron representaron un $46,2 \%$. Conclusión: La mortalidad por COVID-19 alcanzó un 46,20\%; mayores de 65 años (51,90\%), de sexo masculino $(60,40 \%)$ y casados $(65,40 \%)$. Se encontró relación significativa entre factores sociodemográficos como la edad, factores clínicos (comorbilidad), en su mayoría hipertensión arterial, obesidad y diabetes mellitus II, y pacientes con estado de salud reservado con la mortalidad del COVID-19.
\end{abstract}

Palabras Clave: Mortalidad; Covid-19; Factores predisponentes; Enfermedades crónicas; Enfermedades coexistentes Fuente: De(S-BIREME).

\section{ABSTRACT \\ Objetive. To determine the sociodemographic and
clinical risk factors related to mortality in COVID-19 \\ Objetive. To determine the sociodemographic and
clinical risk factors related to mortality in COVID-19 patients in a Hospital in northern Peru. Material and}

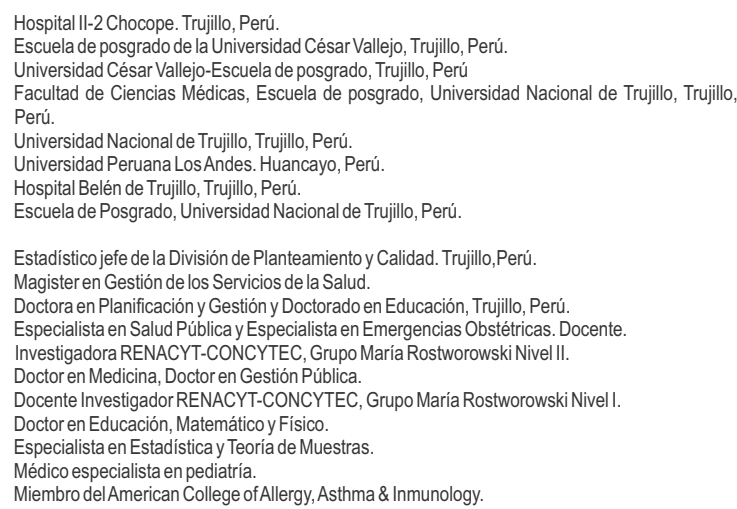

Miembro del American College of Allergy, Asthma \& Inmunology.

Methods: the research was carried out in a Hospital in the north of Peru. The population was made up of 208 Clinical Histories of COVID-19 patients seen between March and July 2020. The independent variable was sociodemographic factors such as age, sex and marital status, and clinical factors such as epidemiological history, risk factors (co-morbidities) and health status and the dependent variable was Covid-19 mortality. Results: COVID-19 mortality reached $46.20 \%$ in patients older than 65 years (51.90\%), male $(60.40 \%)$ and married $(65.40 \%)$, with an epidemiological history (37.5\%) , and risk factors (comorbidity) $74.50 \%$, mainly associated with arterial hypertension (8.2\%), obesity (3.4\%) and diabetes mellitus II $(1.9 \%)$, and indeterminate comorbidities (Others) 6.7\%. There was no mortality in patients whose health status was Stable, while patients with reserved health status and who died represented $46.2 \%$. Conclusion: mortality from COVID-19 reached $46.20 \%$; older than 65 years (51.90\%), male $(60.40 \%)$ and married $(65.40 \%)$. A 
significant relationship was found between sociodemographic factors such as age, clinical factors (comorbidity), mostly arterial hypertension, obesity and diabetes mellitus II, and patients with a reserved health status with the mortality of COVID-19.

Keywords: Mortality; Covid-19; Predisposing factors; Chronic diseases; Coexisting diseases (Source: DeCSBIREME).

\section{INTRODUCCIÓN}

El comportamiento y transición de la mortalidad por COVID-19 a nivel mundial, responde a fuentes y escalas muy diversas y diferentes entre sí, generalmente por lo que están estructuradas de acuerdo a los desarrollos socioeconómicos y políticos de los pueblos y países en los que se analiza o evalúa ${ }^{(1)}$. En aquellos países cuyos censos poblaciones y registros de defunciones han sido más transparente, se han permitido la comparación analítica de la mortalidad en periodos cortos, pudiendo observarse la evolución del fenómeno con mayor precisión y, sobre todo, el comportamiento del mismo según los factores sociodemográficos y clínicos con los cuales se les relaciona ${ }^{(2)}$.

Esta práctica define a la mortalidad como el indicador del comportamiento y transición del COVID-19 en diferentes escalas o alcances. A nivel nacional, departamental o local, los puntos de partida son las fuentes de los registros de defunciones: censo de población y tablas de vida publicados por las instituciones gubernamentales u en el caso de la Unión Europea, por los Censo de Investigación Sociodemográfica y Poblacional, realizados por el Consejo de La Unión Europea. En Estados Unidos, los reportes corresponden a políticas de estados concertados con diversos organismos particulares, en Rusia los reportes del comportamiento y la transición de la mortalidad por covid-19, se realiza según los manejos de los Estados Confederados ${ }^{(3)}$. En países como China, Vietnam, Camboya y la mayoría de países islámicos, los reportes son manejados desde los comités populares o vecinales de base, los mismos que involucran la participación de las mismas comunidades o grupos poblacionales, todos ellos interrelacionados con niveles o centros de información articulados a nivel nacional ${ }^{(4)}$

Los análisis diferenciales de la transición o de los cambios de la intensidad y la estructura de la mortalidad se han realizados a diferentes escalas territoriales $\mathrm{y}$, todos ellos, tienen un punto de vista descriptivos explicativo apoyándose en metodologías estadísticas multivariadas. En la mayoría de países europeos, por ejemplo, la identificación de los factores sociodemográficos y clínicos relacionados la mortalidad del COVID-19 es realizado complementando ajustes del modelo explicativo, es decir, planteando desde un comienzo diferencias encontradas, según periodos de tiempo determinado y dentro de este marco general se analizan las diferencias del impacto de COVID-19, asi como las brechas entre poblaciones. Según esta estrategia se establecen políticas de cercos epidemiológicos para avanzar en la comprensión de cómo se fueron trasformando los procesos de avance de la pandemia hasta llegar a establecerse las diferencias regionales y locales por sexo, grado e instrucción, situación socioeconómica, co-morbilidades clínicas de mayor protagonismo, etc. ${ }^{(5)}$. Esto le permitiría a la mayoría de países de la Unión Europea realizar ajustes en sus modelos de registro y reporte de los datos de mortalidad, además de indicar la forma en que se extendía la pandemia a nivel local, regional (Comunidades Autonómicas) o nacional y la vida y duración del COVID-19 $9^{(6)}$

Hoy en día todos estos procesos no solo son de gran relevancia, sino que se han convertido en una necesidad en todos los países del mundo, especialmente en América Latina donde las tazas estandarizadas de mortalidad por factores sociodemográficos y clínicos, para periodos cortos, es imperativo, ya que es la única manera de procesar los cambios que lentamente se dan en los perfiles epidemiológicos de los hombres y mujeres que sufren la enfermedad dentro de un contexto país ${ }^{(7)}$.

En el Perú y específicamente en la Región La Libertad, los indicadores sobre la mortalidad son inciertos, por la gran cantidad de sub registro que proviene de diferentes fuentes de información, mostrándose que aún no le hemos dado la importancia del caso al comportamiento y avance de la transición de la mortalidad del COVID-19. Con los recursos con que se cuenta, sólo podemos estimar que la mortalidad masculina ha ido siendo mayor que la femenina, pero, que en estos dos últimos meses las brechas de estos indicadores se habrían estrechado de tal manera que no existe un patrón de comportamiento del COVID-19 ni de la transición de la mortalidad en nuestro país. Se señala que en este problema hay una gran concurrencia de factores, siendo la inoperancia y/o paralasis que sufren las instituciones bases o base de la información las principales responsables de ello ${ }^{(8)}$. Es necesario resaltar que los indicadores de la mortalidad distorsiona el perfil epidemiológico de la pandemia y la comprensión de los comportamientos y transición de la mortalidad, a pesar que se hacían esfuerzos por diferenciarla por ejemplo entre poblaciones que presentaban comorbilidades asociadas como la Diabetes Mellitus, cardiopatías congénitas, enfermedades renales, enfermedades hepáticas, hipertensivas, etc., en donde el impacto del COVID-19 se ha configurado como sobremortalidad, especialmente en la población adulta con morbilidades asociadas al COVID-19 ${ }^{(9,10)}$, por lo que no hay una forma de conocer en qué momento el COVID-19 pueda hacer desaparecer la mortalidad por otras causas 
No COVID-19, ni cual seria los motores principales de estos descensos, por lo tanto, las políticas públicas devienen en procedimientos ciegos que pierden rápidamente su protagonismo en la medida que aumenta la mortalidad de algunos grupos poblaciones con o sin co-morbilidades asociadas.

Pero, esta particularidad que ha ocurrido en esta realidad local no es exclusiva, también se ha presentado en países avanzado como España e Italia, incluso en China, en donde, según ${ }^{(11)}$, el comportamiento y transición de la mortalidad del COVID-19 proporcionaba la idea falsa de que el COVID19 sería un virus mutante por naturaleza debido a la confusión inicial en el registro de las muertes, aspecto del trabajo sanitario corregido rápidamente ${ }^{(12)}$. Estos elementos de la realidad mundial, latinoamericana, nacional y local señalan la pertinencia e importancia del estudio, por lo que se planteó como problema a resolver: ¿Cuál es la relación entre los factores sociodemográficos y clínicos con la mortalidad por COVID-19 en un Hospital del norte de Perú? Desde esta perspectiva, el objetivo de esta investigación es determinar los factores de riesgo sociodemográficos como edad y sexo y factores clínicos como la presencia de antecedentes epidemiológicos, factores de riego (co-morbilidades), y estado de salud al momento de internamiento relacionados con la mortalidad en pacientes COVID-19 en un Hospital en el norte de Perú.

\section{MATERIAL Y MÉTODO}

\section{Diseño y Población de Estudio}

Se realizó un estudio descriptivo de relación causal y carácter explicativo. Busca determinan las relaciones de causalidad para explicar procesos predictivos de una variable sobre otra. Cuya variable independiente (Cuantitativa y cualitativa) fueron los factores sociodemográficos con las subvariables como la edad, sexo y el estado civil y los Factores Clínicos cuyas sub variables fueron los antecedentes epidemiológicos, los factores de riesgo (co-morbilidades) y el estado de salud al ingresar y la Variable dependiente (Cuantitativa) fue la mortalidad Covid-19. La población lo constituyeron el universo poblacional de 208 Historias Clínicas de pacientes COVID-19 atendidos entre Marzo a Julio del presente año en un Hospital en el norte de Perú. Se aplicó un muestreo no probabilístico por asignación estratégica considerando al $100 \%$ de la población objeto de estudio. El criterio de Inclusión fueron las Historias clínicas con información completa relativa a los fines de la investigación.

\section{Variables y dimensiones}

Las variables de estudio por su naturaleza fueron cualitativas medidas en escala ordinal y cuantitativa en escala de razón, las dimensiones estudiadas son de alguna manera subvariables y como tales tienen también las escalas de medición, que son base para la interpretación estadística. Los factores sociodemográficos estudiadas son edad, los cuales se establecieron en rangos, la dimensión sexo y estado civil fueron medidas en escala nominal, otra de las dimensiones tratada fueron los factores clínicos, se observó los factores epidemiológicos se aplicó la escala nominal, en factores de riesgo y estado de salud, la escala de medición también fue nominal.

Técnicas e instrumento y procedimiento de los datos La técnica utilizada para aplicar el instrumento fue el Registro de datos en campo con apoyo del sistema informático EsSalud y el Instrumento aplicado en este estudio fue un Protocolo de registro de datos en campo que consto de 3 ítems de filiación y 7 de contenido o de información esencial. Los Procedimientos que se llevaron a cabo en este estudio fue solicitar las autorizaciones correspondientes para extraer información valiosa para el estudio a través del sistema informático institucional en un Hospital en el norte de Perú, para ello se presentaron las solicitudes del caso alcanzando una copia del proyecto de estudio a la institución, encontrando receptividad y apoyo de parte de las autoridades del establecimiento de salud. Una vez conseguidas las autorizaciones para extraer la información relativa al estudio se procedió a coordinar con el feje de la Oficina de Estadística e Informática de la institución coordinando los tiempos y los espacios en que se procedería. La información fue recolectada sin problemas en formato Excel lista para su procesamiento y el tiempo de duración fue de 45 minutos.

\section{Análisis estadísticos}

Los datos fueron trasladados de un formato Excel a un formato de procesamiento estadístico SPSS V22 en español; previamente los datos fueron codificados con códigos numéricos mutuamente excluyentes. La prueba estadística que se uso fue el Chi Cuadrado de Pearso'n ya que solo encontramos datos categóricos dicotómicos (si o no) en los reportes de medición de las variables. En tal sentido no se necesitó para establecer la relación entre las variables pruebas inferenciales. Se estableció que existe relación significativa entre las variables en estudio cuando la significancia estadística presenta una $p<0,05$.

\section{Consideraciones Éticas}

En el presente trabajo se tomó en consideración los principios éticos y/o bioéticos que se estipulan en lo concordado entre OPS y CIOMS, para la realización de investigaciones con la participación de seres humanos. Estos principios declarados en el informe Belmont ${ }^{(13-15)}$ son: Principio de Autonomía, ya que cada uno de los participantes después de la información que recibió sobre los objetivos y fines de la investigación, decidió voluntariamente a participar de ella como encuestado y firmó un formato de consentimiento informado. Además, se respetan los procedimientos éticos exigidos 
por el Comité de Ética de la UCV y la prueba de copia del paquete de información estadística Turnitin.

\section{RESULTADOS}

En la Tabla 1 se presenta la relación entre factores sociodemográficos como edad, sexo y estado civil con la mortalidad del COVID-19 en un Hospital en el norte de Perú, donde se muestra que del total de pacientes COVID-19 atendidos entre marzo a julio del 2020, fallecieron $46,20 \%$. De este grupo, el $30.80 \%$ eran $>65$ años, el 13,90\% con edades comprendidas entre 45 a 65 años y un porcentaje muy bajo (1,40\%) en el rango de edades de 19 a 45 años. Así mismo, los fallecidos de sexo masculino corresponden al $29.80 \%$ y del sexo femenino un $16,30 \%$, en donde el $32,70 \%$ eran casados, un 10.10 $\%$ solteros, $2.90 \%$ viudos y un 0,50 \% divorciados. Así mismo, la prueba de chi-cuadrado de independencia muestra que la edad guarda relación directa $(p \leq 0,05)$ con la mortalidad del COVID-19. De allí que, la mortalidad de COVID-19 se encuentra concentrada en una mayor proporción en pacientes $>65$ años. (Tabla 1 ).

Tabla 1. La relación entre factores sociodemográficos como edad, sexo y estado civil con la mortalidad del COVID-19 en un Hospital en el norte de Perú.

\begin{tabular}{|c|c|c|c|c|c|c|c|}
\hline \multirow{3}{*}{$\begin{array}{c}\text { Factores } \\
\text { sociodemográficos }\end{array}$} & \multicolumn{6}{|c|}{ Mortalidad } & \multirow{3}{*}{$\begin{array}{c}X^{2} \\
\text { P valor }\end{array}$} \\
\hline & \multicolumn{2}{|c|}{ Sí } & \multicolumn{2}{|c|}{ No } & \multicolumn{2}{|c|}{ Total } & \\
\hline & N. ${ }^{\circ}$ & $\%$ & $\mathrm{~N} .^{\circ}$ & $\%$ & N. ${ }^{\circ}$ & $\%$ & \\
\hline \multicolumn{8}{|l|}{ Edad } \\
\hline 4 a 11 años & 0 & 0,00 & 4 & 1,90 & 4 & 1,90 & \\
\hline 11 a 19 años & 0 & 0,00 & 1 & 0,50 & 1 & 0,50 & \\
\hline 19 a 45 años & 3 & 1,40 & 14 & 6,70 & 17 & 8,20 & 0,001 \\
\hline 45 a 65 años & 29 & 13,90 & 49 & 23,60 & 78 & 37,50 & \\
\hline$>65$ años & 64 & 30,80 & 44 & 21,20 & 108 & 51,90 & \\
\hline Total & 96 & 46,20 & 112 & 53,80 & 208 & 100,00 & \\
\hline
\end{tabular}

\begin{tabular}{lccccccc} 
Sexo & & & & & & & \\
Femenino & 34 & 16,30 & 48 & 23,10 & 82 & 39,40 & 0,274 \\
Masculino & 62 & 29,80 & 64 & 30,80 & 126 & 60,60 & \\
\hline Total & 96 & 46,20 & 112 & 53,80 & 208 & 100,00 &
\end{tabular}

\begin{tabular}{lccccccc} 
Estado Civil & & & & & & & \\
Soltero & 21 & 10,10 & 41 & 19,70 & 62 & 29,80 & \\
Casado & 68 & 32,70 & 68 & 32,70 & 136 & 65,40 & 0,064 \\
Viudo & 6 & 2,90 & 2 & 1,00 & 8 & 3,80 & \\
Divorciado & 1 & 0,50 & 1 & 0,50 & 2 & 1,00 & \\
\hline Total & 96 & 46,20 & 112 & 53,80 & 208 & 100,00 &
\end{tabular}

La Tabla 2 muestra que del total de pacientes COVID-19 atendidos entre marzo a julio del 2020 en un Hospital del norte de Perú, un 46,2\% fallecieron, de los cuales un $1,9 \%$ presentaron antecedentes epidemiológicos previos, un $37,5 \%$ presentaron factores de riesgo (comorbilidad), un 1,9\% presentaron Diabetes Mellitus II, un 8,2\% presentaron Hipertensión Arterial, un 3,4\% presentaron Obesidad, mientras que los pacientes que presentaron comorbilidades indeterminadas (Otras) y fallecieron representaron un 6,7\%. De igual manera, no se presentó mortalidad en los pacientes cuyo estado de salud era Estable, mientras que los pacientes con estado de salud reservado y fallecieron representaron un $46,2 \%$ y los que no fallecieron un $46,2 \%$. De esta manera, los resultados de la prueba de chi-cuadrado de independencia evidenciaron que tanto los factores de riesgo, principalmente la hipertensión arterial, obesidad y diabetes mellitus II, presentes en un $74,50 \%$ y el estado de salud (preponderantemente de pronóstico reservado $92,30 \%$ ) fueron los factores clínicos que se relacionaron significativamente $(p \leq$ $0,05)$ con la mortalidad COVID-19.

Tabla 2. La relación entre factores clínicos como antecedentes epidemiológicos, factores de riesgo (comorbilidad) y estado de salud con la mortalidad por COVID-19 en un Hospital del norte de Perú.

\begin{tabular}{|c|c|c|c|c|c|c|c|}
\hline \multirow{3}{*}{ Factores clínicos } & \multicolumn{4}{|c|}{ Mortalidad COVID-19 } & & & \multirow{3}{*}{$\begin{array}{c}\text { X2 } \\
\text { P valor }\end{array}$} \\
\hline & \multicolumn{2}{|c|}{$\mathrm{Si}$} & \multicolumn{2}{|c|}{ No } & \multicolumn{2}{|c|}{ Total } & \\
\hline & $\mathrm{n}^{\circ}$ & $\%$ & $\mathrm{n}^{\circ}$ & $\%$ & $\mathrm{n}^{\circ}$ & $\%$ & \\
\hline \multicolumn{8}{|l|}{$\begin{array}{l}\text { Antecedentes } \\
\text { epidemiológicos }\end{array}$} \\
\hline $\mathrm{Si}$ & 4 & 1,9 & 6 & 2,9 & 10 & 4,8 & \multirow{2}{*}{0,689} \\
\hline No & 92 & 44,2 & 106 & 51,0 & 198 & 95,2 & \\
\hline \multicolumn{8}{|l|}{$\begin{array}{l}\text { Factores de riesgo } \\
\text { (comorbilidad) }\end{array}$} \\
\hline $\mathrm{Si}$ & 78 & 37,5 & 77 & 37,0 & 155 & 74,5 & \multirow{2}{*}{0,039} \\
\hline No & 18 & 8,7 & 35 & 16,8 & 53 & 25,5 & \\
\hline \multirow{2}{*}{\multicolumn{8}{|c|}{ Diabetes Mellitus }} \\
\hline & & & & & & & \\
\hline $\mathrm{Si}$ & 4 & 1,9 & 7 & 3,4 & 11 & 5,3 & \multirow{2}{*}{$<0,05$} \\
\hline No & 92 & 44,2 & 105 & 50,5 & 197 & 94,7 & \\
\hline \multicolumn{8}{|l|}{ Hipertensión } \\
\hline \multicolumn{8}{|l|}{ Arterial } \\
\hline $\mathrm{Si}$ & 17 & 8,2 & 13 & 6,3 & 30 & 14,4 & \multirow[t]{2}{*}{$<0,05$} \\
\hline No & 79 & 38,0 & 99 & 47,6 & 178 & 85,6 & \\
\hline \multicolumn{8}{|l|}{ Obesidad } \\
\hline $\mathrm{Si}$ & 7 & 3,4 & 8 & 3,8 & 15 & 7,2 & \multirow[t]{2}{*}{$<0,05$} \\
\hline No & 89 & 42,8 & 104 & 50,0 & 193 & 92,8 & \\
\hline \multicolumn{8}{|l|}{ Otra Morbilidad } \\
\hline $\mathrm{Si}$ & 14 & 6,7 & 9 & 4,3 & 23 & 11,1 & \multirow[t]{2}{*}{$<0,05$} \\
\hline No & 82 & 39,4 & 103 & 49,5 & 185 & 88,9 & \\
\hline \multicolumn{8}{|l|}{ Estado de Salud } \\
\hline Estable & 0 & 0,0 & 16 & 7,7 & 16 & 7,7 & \multirow[t]{2}{*}{$<0,001$} \\
\hline Reservado & 96 & 46,2 & 96 & 46,2 & 192 & 92,3 & \\
\hline
\end{tabular}

\section{DISCUSIÓN}

Los resultados confirman las tendencias de los reportes internacionales ${ }^{(16)}$ Vences et al sobre la forma como el COVID-19 ataca a las personas según sus edades, la 
presencia de factores de riesgos y el estado de salud con que llegan a ser hospitalizados, sin embargo, estos necesitaban ser confirmados ante la incredulidad de los pobladores para seguir las recomendaciones que hace el Gobierno para evitar el contagio.

Efectivamente, los factores sociodemográficos como el sexo y el estado civil, no tienen relevancia alguna para la mortalidad del COVID-19, pero si la edad ya que está relacionada significativamente con ella, destacándose el hecho que sobre los mayores de 65 años se han dado el $(30,80 \%)$ de las muertes. Estos resultados son consistentes con $^{(16)}$ que en España reporta una media de edad en los fallecidos de 81,50 años con más del $50 \%$ de esta población con comorbilidad asociada al COVID-19. Pero son diferentes a los reportes de Zahang et al en China que si bien es cierto encuentra que la mayoría de paciente fueron personas mayores de 65 años, estos no fallecieron, solo el 25 \% de dicha población falleció por COVID-19. Así mismo, en relación a la presencia de factores de riesgo tales como diabetes mellitus II, hipertensión arterial y obesidad asociados a la mortalidad por COVID-19, los resultados coinciden con los reportados por $^{(17)}$, quienes en un estudio para describir las manifestaciones de pacientes con enfermedad por coronavirus 2019 (COVID-19), evaluaron variables sociodemográficas, antecedentes, manifestaciones clínicas y radiológicas, tratamientos y evolución en pacientes que ingresaron por emergencia, del 6 al 25 de marzo de 2020, al Hospital Nacional Edgardo Rebagliati Martins en Lima, encontraron comorbilidades como hipertensión arterial y obesidad, que se mencionan como factores de riesgo para desarrollar la forma grave de la enfermedad. De igual forma, coinciden con los resultados presentados por ${ }^{(18)}$ en una revisión sobre obesidad como factor de riesgo para mortalidad por COVID-19, entre el 6 de febrero hasta el 17 de mayo del 2020, quienes reportaron que en pacientes con COVID-19, la obesidad fue un factor de riesgo para progresar a mortalidad.

Estos resultados pueden deberse a la secuencia normal del desarrollo biológico de las personas, el mismo que está incorporado en la secuencia de vida de sus propios genes. La secuencia de vida genética de las personas implica un ciclo de vida incluso de las células corporales que prearan al organismo para generar cada vez menos defensas, el adulto mayor, ya no tiene la misma potencia para equilibrar la muerte frente al COVID$19^{(12)}$. Esta situación es potencialmente peligrosa porque las células corporales ya no pueden mantener el funcionamiento principal de los órganos y sistemas del cuerpo, estas funciones tienen un límite genéticamente controlado ya que las células pasan por el mismo proceso del envejecimiento en el cuerpo y posiblemente el COVID-19 encuentra en esta condición la situación perfecta para instalarse con mayor potencia $^{(19)}$.
Así mismo, el reloj biológico de las personas puede actuar a través de genes que controlan cambios hormonales o causan problemas en el sistema inmunológico dejando al cuerpo vulnerable no tan solo ante el COVID-19, sino también, ante otros tipos de enfermedades ${ }^{(20)}$. Está demostrado que con la edad se pierde fuerza muscular, la acumulación de grasa y la atrofia de los órganos están relacionados con la declinación en la actividad hormonal. También hay evidencia de que la eficiencia del sistema inmunológico disminuye y que los niveles de producción de células inmunes producen tazas de sobrevivencia de dos años entre las personas en edad avanzada como es el caso de los mayormente afectados entre la población en estudio $^{(21)}$. La otra explicación es que el reloj biológico de las personas mayores de 65 años en estudio haya estado marcando el paso de una reducción gradual de los telómeros en las puntas protectoras de los cromosomas. Cada vez que las células se dividen se estarían reduciendo más, en ellos, las puntas protectoras ya que su tendencia es que en cierto momento de la vida de las personas estas células detienen su división por falta de telomerasas o enzimas que permiten que las células reparen sus telómeros ${ }^{(22)}$.

El vínculo entre los telómeros más cortos en el ADN en sangre y la muerte temprana por enfermedades cardiacas e infecciosas, por ejemplo, está demostrado desde hace más de 20 años. Por eso es que se recomienda que toda persona mayor de 65 años deba recibir apoyo para que sus células reparen sus telómeros y su cuerpo pueda ejercer un fuerte control de su ciclo de vida y sobre los patrones de envejecimiento. Sin embargo, la programación genética natural no es determinante de manera que todos los seres humanos no pueden morir a la misma edad, pero, si son afectos incluso a factores ambientales u virales de todo tipo ${ }^{(12)}$.

Las teorías de taza variables denominada las teorías del error, consideran el envejecimiento como resultado de procesos que varía de una persona a otra y, son influidos por factores internos y externos. En la mayoría de las teorías de la tasa variable, el envejecimiento involucra daño debido a errores fortuitos o por agresiones ambientales a los sistemas biológicos. Otras se concentran en los procesos internos como el metabolismo que es un proceso por el cual el cuerpo convierte al alimento y el oxígeno en energía. Se estima que el metabolismo influye de manera directa y continua en la tasa de envejecimiento. La teoría del desgaste, sostiene que el cuerpo envejece como resultado del daño acumulado por el sistema, más allá de la capacidad del cuerpo para repararlo ${ }^{(23)}$.

Es decir, desde el punto de vista de estas teorías, el COVID-19 sería el que genera un daño que no puede ser manejado por el cuerpo humano en condiciones de envejecimiento ya que, a medida que las células 
envejecen son menos capaces de reparar o reemplazar los componentes dañados. Esto genera estresores internos que junto a estresores externos pueden agravar el proceso de desgaste ${ }^{(24)}$.

Sin embargo, hay una teoría que es más precisa para temas como la agresividad del COVID-19 porque se centran en los efectos nocivos de los radicales libres: átomos o moléculas de oxigeno sumamente inestable por la intervención del virus del COVID-19. Estos radicales libres son formados durante el metabolismo y reaccionan y pueden dañar las membranas celulares, proteínas celulares, las grasas, los carbohidratos, incluso el ADN. El daño que produciría el COVID-19 sería como un radical libre en el oxígeno y se acumularía con mayor rapidez en personas de edad avanzada ya que en estas personas se les asocia el fenómeno con problemas de artritis, distrofia muscular, cataratas, cáncer, diabetes de inicio tardía y trastornos neurológicos como la enfermedad del Parkinson ${ }^{(11)}$.

En China ya se han desarrollado estudios que apoyan la teoría del COVID-19 como productor de radicales libres en los átomos moleculares del oxígeno, también han encontrado evidencia de que es capaz de mutar en el $A D N$ de las mitocondrias que envejecen y que generan energía en las células humanas, lógicamente que también se está demostrando aquí su papel como productor de radicales libres en este nive ${ }^{(25)}$.

Aunque las investigaciones actuales siguen su curso, los efectos de los suplementos antioxidantes que supuestamente podrían contrarrestar la actividad del COVID-19 como productor de radicales libres, no son concluyentes ya que no ha podido descifrar a plenitud los códigos genéticos del ADN del virus, de allí que China, Rusia y Corea del Norte, recomiendan la ingesta de grandes cantidades de vitamina $C$ y vegetales con altos contenidos en beta caroteno, al parecer ayuda a proteger o evitar la actividad del COVID-19, para generar radicales libres en las células de oxígeno que requiere el organismo ${ }^{(21)}$.

Estos resultados que pueden estar evidenciando dos cosas: Falta de pericia y capacidad para atender los requerimientos de la atención y/o, el acceso tardío de los pacientes al servicio, ya que solo tendrían acceso a los servicios cuando la enfermedad está muy avanzada $^{(8)}$.

Sobre la primera hipótesis tendríamos que la falta de pericia y capacidad para atender pacientes COVID-19, seria relativamente cierta, no por falta profesionalismo del personal, sino, por la concurrencia de un conjunto de factores que distorsionan su accionar corporativos. Tiene una gran cantidad de obligaciones para protegerse el mismo y prevenir la transmisión en ambientes hospitalarios, que muchas veces resulta tenebroso y penoso ver que no se puede accionar sin pensar dos veces en las consecuencias a nivel personal y familiar, ya que estamos hablando de un trabajo de alto riesgo, que ha quitado la vida a más del $20 \%$ de profesionales involucrados en su atención ${ }^{(26)}$.

Su preocupación empieza en la forma como debe implementarse apropiadamente para evitar el contagio, esto implica que deba estar capacitada en cómo colocar, trabajar y quitarse el EPP, pasar por los controles, asegurar la disponibilidad de recursos: tratamientos, facilidad de acceso a las pruebas de laboratorio, la formación clara consignada en las Historias clínicas, revisar los protocolos de control ambiental, etc., pero, sobre todo que ningún personal de salud ha podido hasta la fecha ser capacitado adecuadamente para la atención de pacientes COVID19. Es más, el comportamiento errático y variado de la enfermedad, sus formas de manifestarse y de cambiar las condiciones de salud de las personas, son muy poco manejables y hasta impredecibles en la mayoría de los $\operatorname{casos}^{(27)}$.

Entrando en el debate sobre los desafíos y consecuencias de la pandemia y la forma en que se construyen las respuestas y usos de las capacidades profesionales en la atención, el primer desafío es encontrar en la implementación de medidas de protección un contexto adecuado y sobre todo de claridad en los procedimientos a seguir. No basta con la intuición del profesional, sino también de saber quehacer en determinadas situaciones dentro del contexto de la atención del paciente propiamente dicho ${ }^{(28)}$.

Esta situación refleja que el avance de las medidas para profundizar el control y reducción de la mortalidad por COVID-19 es un proceso en curso muy difícil de determinar por cuanto de la transmisión del virus y sus comportamientos como cuadro clínico, aun se conoce la punta del iceberg y se ve acompañada por una gran incertidumbre sobre el quehacer con ella ${ }^{(19)}$.

Los procesos para atender las emergencias demuestran profundas prácticas de transformación del trabajo diario del profesional de salud y, muchas veces las rutinas seguidas antes del COVID-19 son difíciles de ser dejadas de lado a pesar que el profesional de salud ha demostrado una gran capacidad para adaptarse a las nuevas condiciones del trabajo. Esto, por supuesto que escapa al ojo de quien juzga desde fuera, pero es entendible que estos elemento puedan influencias en contra de un accionar corporativo ideal ${ }^{(28)}$.

Por otro lado, el llegar demasiado tarde a la atención puede también responder a la concurrencia de varios factores entre ellos a las nuevas prácticas sociales que se reproducen en la vida cotidiana. Tenemos que pensar en la posibilidad de construir sociedades más solidarias que estén mejor implementadas y dotadas para no ver 
nuevamente la forma de decirles a los pacientes que no hay cupos para la hospitalización o su internamiento oportuno. Al parecer el problema no está en el paciente está en la incapacidad del sistema sanitario para atender la gran demanda de atención ${ }^{(29)}$.

Los sistemas de salud se colapsan en todo el mundo, las razones económicas son simplemente obvias, por lo que paradójicamente es la clave que reduce las interacciones sociales para resolver de manera directa el tema del acceso oportuno del paciente a la atención. Construir alternativas creativas en ese campo es uno de los retos para la imaginación y la intuición, sobre cómo en una intervención continúa utilizando el estado de exención para poner en práctica estrategias de reducción de la necesidad del usuario de utilizar los servicios hospitalarios. Es decir regresamos al punto inicial en la que tendríamos que proponer el evitar que el paciente se infecte por la enfermedad, especialmente los que saben que están afectos a otras patologías y se encuentran dentro de las poblaciones etarias en vulnerabilidad ${ }^{(9)}$.

La idea no es nunca descabellada, pero requiere de un trabajo que no está al alcance la intervención de los profesionales de salud que atienden al paciente COVID19. Es decir son elementos externos de la atención que condicionan su sestado de salud y determinan su riesgo de morir por COVID-19 ${ }^{(29)}$. Frente a ello se potencia la idea que el personal de salud de todas las instituciones sanitarias necesita de la cooperación del conjunto de las interacciones sociales para evitar mayores índices de mortalidad. La Reflexión debe girar sobre cómo pactar la discusión hasta priorizar la salud de la economía o la salud de la población. En ese sentido, es posible analizar las alternativas sociales para disminuir los niveles de mortalidad que produce COVID-19 ${ }^{(29)}$.

Como consecuencia del análisis de los resultados, llegamos a las siguientes conclusiones: La mortalidad COVID-19 alcanzó al 46,20 \% de pacientes atendidos entre marzo a julio del 2020 en un Hospital del norte de Perú. La mayoría de pacientes fueron mayores 65 años $(51,90 \%)$, de sexo masculino $(60,40 \%)$ y casados $(65,40 \%)$. Los pacientes con antecedentes epidemiológicos representaron solo un $37,5 \%$, los que presentaron factores de riesgo (comorbilidad) un $(74,50 \%)$, principalmente asociados con hipertensión arterial $(8,2 \%)$, obesidad $(3,4 \%)$ y diabetes mellitus II $(1,9 \%)$, mientras que los pacientes que presentaron comorbilidades indeterminadas (Otras) y fallecieron representaron un 6,7\%. De igual manera, no se presentó mortalidad en los pacientes cuyo estado de salud era Estable, mientras que los pacientes con estado de salud reservado y fallecieron representaron un $46,2 \%$ y los que no fallecieron un $46,2 \%$. Finalmente se encontró una relación directa entre los factores de riesgo sociodemográficos como la edad, así como con los factores de riesgo clínicos (comorbilidad), siendo los más frecuentes la diabetes mellitus II, hipertensión arterial y obesidad y los pacientes cuyo estado de salud era reservado con la mortalidad del COVID-19.

Conflictos de interés: Los autores niegan conflictos de interés.

Financiamiento: El estudio no contó con financiamiento debido a que se usó una base de datos ya existente.

\section{REFERENCIAS BIBLIOGRÁFICAS}

1. Herrera Ramírez D. ¿Enfrentar el COVID 19 o aprender de su embate? Práctica Familiar Rural. 2020; 5(1): p. 184.

2. Gro Harlem B, Elhadj As S. Cómo prevenir la próxima pandemia. Planeta Futuro. El Pais. Enero 2020.

3. Charts C. Worldometer. [Internet]. 2020. [citado el 28 de Marzo 2020]. Disponible en: https://www.worldometers.info/coronavirus/?ut m campaign=homeAdUOA?Si.

4. Xiao E. The Japan times. [Internet]. 2020 [citado el 8 de Febrero de 2020]. Disponible en: https://www.japantimes.co.jp/news/2020/02/0 8/asia-pacific/science-health-asia-pacific/chinatapping-ai-big-data-get-grip-coronavirusoutbreak/\#.XnVuaaj7SUk.

5. Leach M. Echoes of Ebola: social and political warnings for the COVID-19 response in African settings. [Internet]. 2020 [citado el 6 de Marzo de $\left.\begin{array}{llllll}2 & 0 & 2 & 0\end{array}\right]$. D is pon ible e n : http://somatosphere.net/forumpost/echoes-ofebola/.

6. Slavoj Z. Zizek: El coronavirus es un golpe a lo 'Kill Bill' al capitalismo. [Internet]. 2020 [citado en Febrero 2020 ]. Disponible en: https://www.climaterra.org/post/zizek-elcoronavirus-es-un-golpe-a-lo-kill-bill-alcapitalismo.

7. Pineda E. Coronavirus: por qué todo México debe actuar ya. [Internet]. 2020. [citado el 16 de Marzo d e 2020 . Dis pon ible e n : https: / / www. nexos.com. $\mathrm{mx} /$ ? $\mathrm{p}=47352$.

8. OPS/CEPAL. América Latina y el Caribe ante la pandemia del COVID-19: Efectos económicos y sociales. Primera ed. Santiago: CEPAL; 2020.

9. Wise T, Zbozinek D, Michelini G, Hagan , Mobbs D. Cambios en la percepción del riesgo y el comportamiento protector autoinformado durante la primera semana de la pandemia de COVID-19 en los Estados Unidos. The Royal Society. 2020; 16; 7(9).

10. Stiff C. La teoría de juegos de la compra por pánico y cómo reducirla. The Conversation. Marzo 2020.

11. Zhang J, Wang X, Jia , Li J, Hu , Chen G, et al. Factores de riesgo para la gravedad, la no mejora y la mortalidad de la enfermedad en pacientes con 
COVID-19 en Wuhan, China. CMI - Clinical Microbiology and Infection. 2020 Junio 1; XXVI(6): p. 767-772.

12. Andersen KG, Rambaut A, Lipkin , Holmes, Garry R. El origen proximal del SARS-CoV-2. Nature Medicine. 2020; 17; p. 450 - 452.

13. Asociación Médica Mundial. Declaración de Helsinki de la Asociación Médica Mundial Principios éticos para las investigaciones médicas en seres humanos 64 Asamblea General. Asociación Médica Mundial. Octubre 2013.

14. (CIOMS) CdOIdICM, Organización Panamericana de la Salud (OPS). Pautas éticas internacionales para la investigación relacionada con la salud con seres humanos. Elaboradas por el Consejo de Organizaciones Internacionales de las Ciencias Médicas (CIOMS) en colaboración con la Organización Mundial de la Salud (OMS). Organización Panamericana de la Salud (OPS) y Consejo de Organizaciones Internacionales de las Ciencias Médicas (CIOMS). Diciembre 2016.

15. Observatori de Bioética I Dret. Informe de Belmont Principios y Guías Éticos para la protección de los sujetos humanos de investigación. Observatori de National Institutes of Health. Abril 1979.

16. Poblador Plou B, Carmona Pírez J, loakeim Skoufa I, Poncel Falcó A, Bliek Bueno , Cano del Pozo M, et al. Comorbilidad crónica y mortalidad basales en casos COVID-19 confirmados por laboratorio: resultados del estudio PRECOVID en España. Revista Internacional de Investigación Ambiental y Salud Pública. 2020; XVII(14).

17. Acosta G, Escobar G, Bernaola G, Alfaro J, Taype W, Marcos C, Amado J. 2020. Caracterización de pacientes con covid-19 grave atendidos en un hospital de referencia nacional del Perú. Revista Peruana de Medicina Experimental y Salud P u blica. $37(2)$ : $253-258$. https://doi.org/10.17843/rpmesp.2020.372.543 7

18. Tenorio-Mucha J, Hurtado-Roca Y. 2020. Revisión sobre obesidad como factor de riesgo para mortalidad por COVID-19. Acta Médica Peruana. 37(3): 324-329. https://doi.org/10.35663/ amp.2020.373.1197

19. Shim E, Tariq A, Choi , Lee Y, Chowell. Potencial de transmisión y gravedad del COVID-19 en Corea del Sur. Revista Internacional de Enfermedades Infecciosas. 2020; 93: 339-344.

20. Chen N, Zhou M, Dong, Qu J, Gong, Han Y, et al. Epidemiological and clinical characteristics of 99 cases of 2019 novel coronavirus pneumonia in Wuhan, China: a descriptive study. The Lance Regional Health. 2020; 395: 507-513.

21. Zhao, Zhong, Xie, Yu Q, Liu J. Relation Between Chest CT Findings and Clinical Conditions of Coronavirus Disease (COVID-19) Pneumonia: A Multicenter Study. PubMed. 2020; 214(5): 10721077.

22. Huang, Wang Y, Li X, Ren L, Zhao J, Hu Y, et al. Clinical features of patients infected with 2019 novel coronavirus in Wuhan, China. The Lancet. 2020; 395: 497-506.

23. Vélez M, Velásquez Salazar P, Acosta Reyes J, Vera Giraldo, Santiago Franco , Jiménez, et al. Clinical factors for the prognosis of severe illness and death in patients with COVID-19. Universidad de Antiquia - Unidad de Evidencia y Deliberación para la Toma de Decisiones-UNED. 2020: 9-15.

24. Zhen Zhang Y, Holmes C. A Genomic Perspective on the Origin and Emergence of SARS-CoV-2. Science Direct Cell. 2020; 181: 223-227.

25. Resources) I(oGSaN. COVID-19 Knowledge and Data Hub: Chinese Academy of Sciences 2020. [Internet]. 2020 [citado el 12 de Marzo de 2020. Disponible en: http://geodoi.ac.cn/covid19/en/index.aspx.

26. Ahmad T, Khan M, Khan FM, Huia J. ¿Are we ready for the new fatal Coronavirus: scenario of Pakistan? Human Vaccines and Immunotherapeutics. 2020; 16(3): 736-738.

27. Liu S, Yang L, Zhang, Xiang YT, Liu Z, Hu, et al. Servicios de salud mental en línea en China durante el brote de COVID-19. The Lancet Psychiatry. 2020; VII(4): E17-E18.

28. Xiang YT, Jin Y, Wang, Zhang, Zhang L, Cheung T. Tribute to health workers in China: A group of respectable population during the outbreak of the COVID-19. International Journal of Biological Sciences. 2020; XVI(10): 1739-1740.

29. Lunn P, Belton C, Lavin , McGowan F, Timmons S, Robertson. Uso de la ciencia del comportamiento para ayudar a combatir el coronavirus. Journal of Behavioral Public Administration. 2020; III(1).

\section{Correspondencia}

Miryam Lora Loza.

Correo: mlora@ucv.edu.pe

Revisión de pares

Recibido: 04/12/2020

Aceptado: 20/12/2020 